\title{
How to Diagnose and Manage Systemic Autoinflammatory Diseases in Childhood
}

\author{
Hanna Bonnekoh, MSC ${ }^{1}$ \\ Karoline Krause, $M D^{1,2, *}$
}

\author{
Address \\ *,1Department of Dermatology and Allergy, Allergie-Centrum-Charité, \\ Charité-Universitätsmedizin Berlin, Charitéplatz 1, 10117, Berlin, Germany \\ Email: karoline.krause@charite.de \\ ${ }^{2}$ Autoinflammation Reference Center Charité $\left(A R C^{2}\right)$, Charité-Universitätsmedizin \\ Berlin, Berlin, Germany
}

Published online: 1 July 2015

(C) Springer International Publishing AG 2015

This article is part of the Topical Collection on Pediatric Dermatology and

Allergy

Keywords Autoinflammatory disease - Cryopyrin-associated periodic syndrome $\cdot$ Hereditary periodic fever .

Interleukin-1 $\cdot$ Interleukin-6 • Systemic juvenile idiopathic arthritis • Urticaria

\section{Opinion statement}

Systemic autoinflammatory diseases (sAIDs) are severe disabling conditions associated with a significant morbidity and limited awareness. Cryopyrin-associated periodic syndrome (CAPS) and systemic juvenile idiopathic arthritis (sJIA) are two prototype sAIDs that present with chronic urticarial or maculo-papular rash in early childhood. A diagnosis of SAID relies on combined clinical symptoms, a positive family history, where applicable, and typical laboratory results. Treatment with anti-cytokine blockers targeting interleukin-1 (IL-1) or IL-6 is effective in reducing clinical symptoms and inflammatory markers and approved for the treatment of CAPS and/or sJIA. In order to prevent severe complications such as amyloidosis, destructive arthropathy or macrophage activation syndrome (MAS), it is important to diagnose patients early and to initiate anti-IL-1 blockade in symptomatic CAPS patients and anti-IL-1 or anti-IL- 6 blockade in therapyrefractive sJIA patients without delay.

\section{Introduction}

Systemic autoinflammatory diseases (sAIDs) belong to a heterogeneous group of rare and severe chronic inflammatory disorders. The term "autoinflammatory disease" was first coined by McDermott et al. following the discovery of the mutation responsible for the tumor- necrosis-factor-receptor-associated periodic syndrome (TRAPS) [1]. In contrast to autoimmune diseases, hightiter autoantibodies and antigen-specific $\mathrm{T}$ cells are usually absent in sAIDs. The uncontrolled inflammation is caused by disturbances in innate immune pathways and 
results in an overwhelming cytokine release. Clinically, patients with sAIDs present with a wide range of symptoms including recurrent fever, fatigue, gastrointestinal, neurologic, musculo-skeletal and dermatologic manifestations. As skin eruptions like urticarial or maculo-papular exanthema are frequent findings in sAIDs, dermatologists and allergists should be aware of these rare diseases and incorporate them in their differential diagnosis [2•]. The majority of disorders start in early childhood and present with considerable quality of life (QoL) impairment $[3 \bullet 4$ ] . Long-term complications include growth and mental retardation and irreversible or- gan damage such as deafness and amyloid-induced renal failure [5]. Within the last decade, a growing number of hereditary monogenic and acquired multifactorial sAIDs have been identified. Among them are many so-called inflammasomopathies with disturbances in IL-1 $\beta$ production and signalling. Since the introduction of very effective anti-cytokinetargeted therapies, the daily life and long-term prognosis

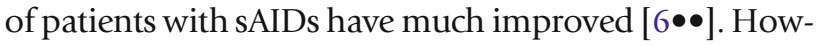
ever, the awareness of sAIDs is still limited and diagnostic delay of years or even decades may occur [7]. We here give an overview on the diagnosis and recent advances in the management of sAIDs in childhood.

\section{Classification of systemic autoinflammatory diseases}

SAIDs can be classified according to the affected signalling pathway or clinical phenotype. For practical reasons, sAIDs are divided into early-onset monogenic disorders associated with a single disease-causing mutation and multifactorial acquired disorders of variable onset and unknown cause. Within others, monogenic sAIDs include the well-known hereditary periodic fever syndromes (HPFs), namely familiar Mediterranean fever (FMF), mevalonate kinase deficiency (MKD), cryopyrin-associated periodic syndrome (CAPS) and TRAPS. Among the multifactorial acquired diseases that manifest during childhood are systemic juvenile idiopathic arthritis (sJIA) and periodic fever, aphthous stomatitis, pharyngitis and adenitis (PFAPA) syndrome. Notably, disturbances in innate immune pathways and autoinflammatory mechanisms are not restricted to very rare sAIDs but can be found as contributing factors in frequent inflammatory disorders such as Crohn's disease, gout and diabetes mellitus too [8-10].

\section{Systemic autoinflammatory diseases with relevance for dermatologists and allergists}

Chronic recurrent wheals are among the most frequent clinical symptoms presented to dermatologists and allergists in daily practice. In the vast majority, chronic urticaria can be diagnosed. However, it is important to not miss other rare causes of recurrent wheals such as autoinflammatory diseases [11].

\section{Cryopyrin-associated periodic syndrome}

CAPS represents a rare hereditary autosomal disease entity with an estimated frequency of one to three cases per million people [12]. So far, most affected families and sporadic cases have been diagnosed in North America and Western European countries, but case reports from other descents also exist [7]. CAPS is considered a disease spectrum of three clinical subtypes: familial cold autoinflammatory syndrome (FCAS) as the mildest form, followed by the 
intermediate subtype Muckle-Wells syndrome (MWS) and the most severe form neonatal-onset multisystemic inflammatory disease, also known as chronic infantile neurological, cutaneous and articular (NOMID/CINCA) syndrome. Overlap between the subtypes may occur [13], and disease severity ranges from mild daily symptoms and few disease attacks in FCAS to continuous inflammation and severe illness with subsequent organ damage in NOMID. Disease onset usually starts in infancy or early childhood but can also be in adolescence [12]. Clinically, CAPS patients present with cold-induced or spontaneous urticarial rash (Fig. 1). In addition, recurrent fever episodes, malaise, joint (arthralgia, destructive arthropathy) and ocular (conjunctivitis, uveitis) involvement and neurologic (headache, sterile meningitis) manifestations may occur. In FCAS and MWS, cold temperatures are a major disease trigger that induces urticarial rash, fever and other systemic symptoms. Long-term complications include deafness and AA amyloidosis with subsequent renal failure.

The chronic inflammation in CAPS is caused by a gain of function mutation in the gene encoding for nucleotide-binding-like receptor protein 3 (NLRP3) $[14,15]$. NLRP3 belongs to a cytoplasmatic multi-protein complex, the inflammasome, that processes pro-interleukin-1 $\beta$ (pro-IL-1 $\beta$ ) and pro-IL-18 [16]. As a consequence of the NLRP3 overactivation, IL-1 $\beta$ and IL-18 are massively secreted which results in systemic inflammatory symptoms as mentioned above $[17,18]$.

\section{Systemic juvenile idiopathic arthritis}

Juvenile idiopathic arthritis (JIA) is the most frequent rheumatic disease in children with a prevalence of 16-150 cases per 100,000 inhabitants [19] and comprises a heterogeneous spectrum of chronic inflammatory and autoimmune disorders. Systemic juvenile idiopathic arthritis (sJIA), also known as Still's disease, represents the most severe JIA subtype and accounts for 5-10 \% of JIA

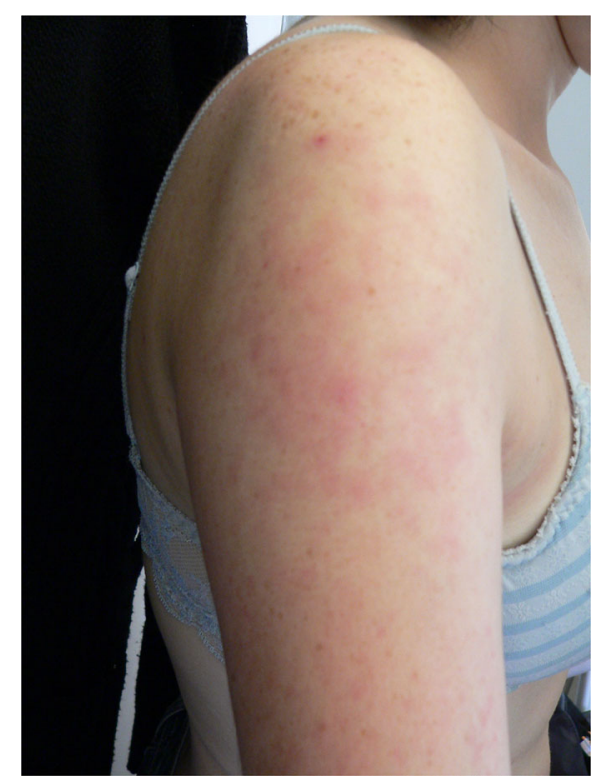

Fig. 1. Urticarial rash in adolescent girl with CAPS (FCAS phenotype). 
cases in Europe (up to $50 \%$ in Japan) [20-22]. The preferred age of onset lies between 1 and 5 years [23]. Clinical symptoms in sJIA are intermittent high-grade fever, arthritis, salmon-coloured maculo-papular or urticarial exanthema, lymphadenopathy, hepatosplenomegaly and serositis [24]. The clinical disease course distinguishes between monophasic presentation with complete resolution of symptoms (ca. $40 \%$ ), chronic remittent disease with active and symptom-free episodes (ca. $10 \%$ ) and chronic active disease with progressive joint destruction (ca. $50 \%$ ) [25]. A feared acute complication in sJIA is macrophage activation syndrome (MAS) that affects around $10 \%$ of patients and goes along with high fever, cytopenia, impaired liver function, coagulopathy and considerable mortality [26]. Long-term complications also include AA amyloidosis [27, 28].

Although its pathophysiology is mainly unknown, sJIA is considered a multifactorial acquired sAID. In contrast to other JIA subtypes, sJIA patients do not show human leukocyte antigen (HLA) associations or autoantibodies [24] but upregulation of IL-1-related cytokines (IL-1 $\beta$, IL-6, IL-18) suggesting an inflammasome-mediated pathogenesis [29-32].

\section{Diagnosing systemic autoinflammatory diseases in childhood}

In children presenting with chronic urticarial or maculo-papular rash and systemic symptoms such as episodic fever, malaise and joint pain of unknown origin, sAIDs should be explored as a rare differential diagnosis. Usually, the diagnosis is established on the basis of combined clinical symptoms, a positive family history (where applicable) and laboratory results [11].

Recently, the Paediatric Rheumatology International Trials Organisation (PRINTO) developed and validated a set of provisional clinical criteria for the diagnosis and classification of HPFs in 1215 consecutive patients [3•]. For CAPS, the existence of urticarial rash, conjunctivitis and progressive hearing loss were identified as the three main clinical criteria that distinguish CAPS from other HPFs [3•]. A normal hearing function, however, does not exclude CAPS, as FCAS patients and many children with MWS and NOMID do not or not yet show impaired hearing function. The urticarial rash in CAPS is symmetrically distributed on trunk and extremities, shows no or little pruritus and does not respond to antihistamine treatment [2•]. Arthralgia is reported in $92 \%$ of cases with CAPS, other clinical symptoms show higher variability [3•].

According to the "International League of Associations for Rheumatology" (ILAR), a diagnosis of sJIA relies on the clinical criteria high-grade quotidian fever for $\geq 3$ consecutive days over 2 weeks or more, arthritis and at least one of the following symptoms in children $<16$ years: evanescent rash, generalized lymphadenopathy, hepatosplenomegaly and serositis [33]. temic symptoms has been reported in around $60 \%$ of cases [3•]. A 
negative family history suggests spontaneous mutations and is frequently seen in NOMID patients [34]. In contrast, familiar cases in sJIA are unusual.

Typically, autoinflammatory patients present with elevated inflammation markers C-reactive protein (CRP), erythrocyte sedimentation rate (ESR) and serum amyloid A (SAA) during disease attacks of acute inflammation. Also, S100 proteins S100A8/9 and S100A12 have been shown to be sensitive markers of disease activity and may detect even subclinical inflammation in sAIDs [35, 36]. Further non-specific laboratory findings are inflammation-induced anaemia, leukocytosis and thrombocytosis [19]. Histopathology from lesional skin of urticarial autoinflammatory diseases like CAPS and sJIA reveals neutrophil-rich dermal infiltrates [37-39].

The diagnosis of CAPS is confirmed by a heterozygous mutation in the NLRP3 gene. Notably, up to $40 \%$ of patients with typical clinical CAPS phenotype are mutation negative suggesting the existence of other yet unknown genetic abnormalities [34]. In a subgroup of mutation-negative patients, genetic mosaicisms were recorded $[34,40]$.

For sJIA, a multifactorial acquired disorder, no genetic tests are available. Genetic association studies revealed no abnormalities in genes of known monogenic sAIDs; nevertheless, genetic polymorphisms in pro- and anti-inflammatory cytokine pathways (e.g. IL-1, IL-6, IL-10) have been reported $[32,41]$. In addition, serum ferritin levels are found to be highly elevated (up to ten times above upper limit of normal) in most sJIA patients and may serve as diagnostic biomarker discriminating sJIA from CAPS and other chronic inflammatory disorders $[42 \bullet \bullet]$.

Differential diagnosis

Clinical symptoms such as urticarial rash, fever and joint pain are not only observed in CAPS and sJIA but may also occur in many other disorders during childhood. Depending on the leading clinical symptoms, dermatologic, hematologic, infectious, autoimmune and other autoinflammatory conditions have to be excluded (Table 1 ).

\section{Management of systemic autoinflammatory diseases}

SAIDs are severe chronic inflammatory diseases that may persist for life and/or lead to irreversible long-term complications such as amyloidosis, destructive arthropathy and deafness. Also, sAIDs are associated with considerable mortality due to acute MAS or amyloid-induced renal failure. In order to improve clinical symptoms and QoL, to prevent long-term complications and to inhibit damage progression, the treatment of sAIDs should focus on controlling the aberrant inflammation. 


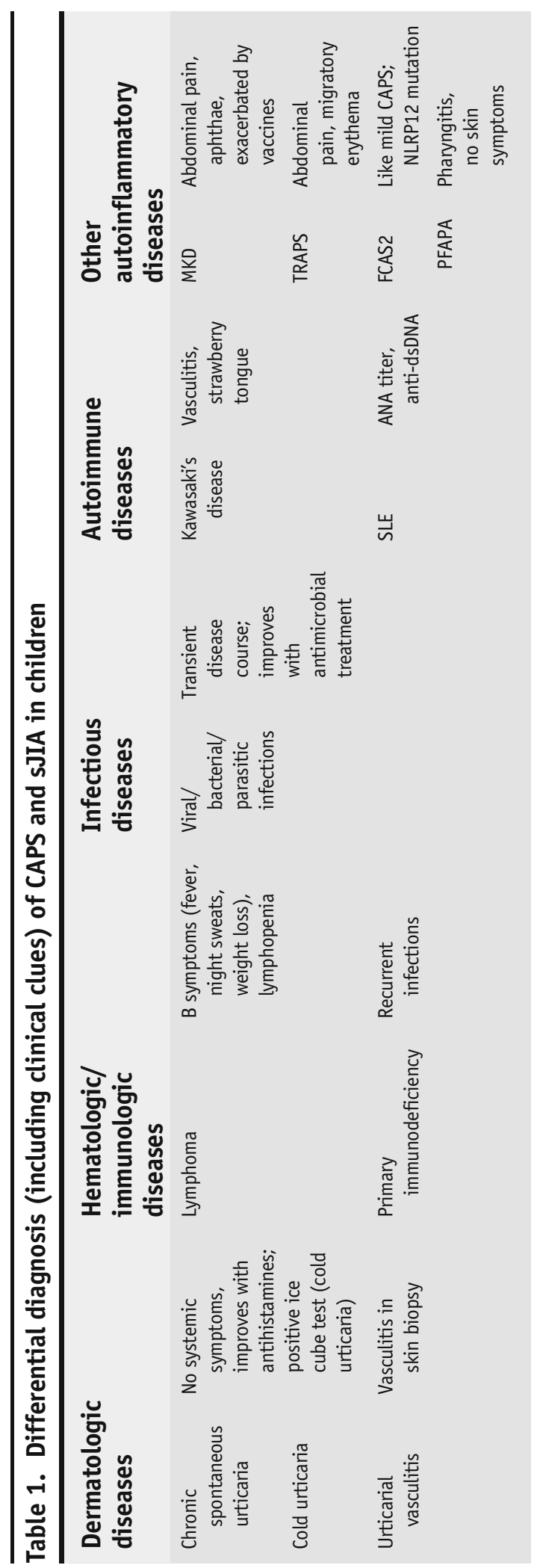




\section{Pharmacologic treatment options in CAPS}

Before the availability of anti-cytokine-targeted therapies, treatment in CAPS was limited to poorly effective corticosteroids, non-steroidal antiphlogistics and immunomodulating drugs.

The first evidence of high efficacy of anti-IL-1-targeted drugs in CAPS derived from a case report of two MWS patients with amyloidosis who showed rapid clinical and inflammatory response within hours following the administration of the IL-1 receptor antagonist anakinra [43]. To date, three different anti-IL-1 blockers are available for the use in CAPS: the recombinant IL-1-receptor (IL-1R) antagonist anakinra (Kineret ${ }^{\circledR}$ ), the fusion protein rilonacept (Arcalyst ${ }^{\circledR}$ ) consisting of the extracellular fraction of the IL-1-R and an IL-1-R accessory protein and the fully human monoclonal IL- $1 \beta$ antibody, named canakinumab (Ilaris $^{\circledR}$ ) (Table 2). All three anti-IL-1 blockers are administered by subcutaneous injections in daily (anakinra), weekly (rilonacept) or 8-weekly (canakinumab) intervals depending on their individual half-life. Placebocontrolled studies with anakinra, rilonacept and canakinumab in children and adults of all CAPS phenotypes demonstrated complete clinical and laboratory response in the majority of patients and led to EMA and FDA approval for this indication [44-46]. Anti-IL-1 blockade also showed to improve long-term complications such as progressive hearing loss and amyloidosis in MWS and NOMID patients [47-49].

Common side effects of anti-IL-1 treatment in CAPS include painful injection site reactions (in particular seen with anakinra), respiratory tract infections and vertigo [44, 45]. Also, a transient increase in transaminases, elevated cholesterol levels and neutropenia may occur [50]. Based on its good efficacy and favourable safety profile, anti-IL-1 blockade is the treatment of choice in symptomatic CAPS patients.

\begin{tabular}{|c|c|c|c|c|}
\hline & Anakinra & Rilonacept & Canakinumab & Tocilizumab \\
\hline Mechanism & 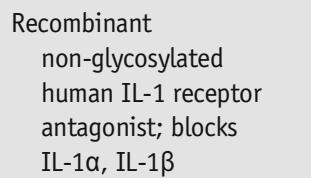 & $\begin{array}{l}\text { Dimeric glycoprotein } \\
\text { (IL-1 Trap); blocks } \\
\text { IL-1 } \alpha, \text { IL-1 } \beta, \text { IL-Ra }\end{array}$ & $\begin{array}{l}\text { Fully human, selective anti-IL-1 } \beta \\
\text { monoclonal antibody; blocks } \\
\text { solely IL-1 } \beta\end{array}$ & $\begin{array}{l}\text { Humanized monoclonal anti-IL-6 } \\
\text { receptor antibody; blocks } \\
\text { soluble and membrane-bound } \\
\text { IL-6receptor }\end{array}$ \\
\hline Half-life & $4 \mathrm{~h}$ & $67 \mathrm{~h}$ & $21-28$ days & up to 23 days \\
\hline $\begin{array}{l}\text { Dosing regime } \\
\text { in children }\end{array}$ & $\begin{array}{l}\text { CAPS: } \\
1-2 \mathrm{mg} / \mathrm{kg} \text { s.c., } \\
\text { daily injections }\end{array}$ & $\begin{array}{l}\text { CAPS: } \\
\text { Loading dose } 320 \mathrm{mg} \\
\text { s.c., followed by } 160 \mathrm{mg} \\
\text { weekly injections }\end{array}$ & $\begin{array}{l}\text { CAPS: } \\
2 \mathrm{mg} / \mathrm{kg} \text { in } \geq 15 \mathrm{~kg}, \\
4 \mathrm{mg} / \mathrm{kg} \text { in } 7.5-15 \mathrm{~kg} \text { s.c. } \\
\text { injections every } 8 \text { weeks } \\
\text { sJIA: } \\
4 \mathrm{mg} / \mathrm{kg} \text { in } \geq 7.5 \mathrm{~kg} \text { s.c. injections } \\
\text { every } 4 \text { weeks }\end{array}$ & $\begin{array}{l}\text { sJIA: } \\
8 \mathrm{mg} / \mathrm{kg} \text { in } \geq 30 \mathrm{~kg}, 12 \mathrm{mg} / \mathrm{kg} \\
\text { in }<30 \mathrm{~kg} \text { i.v. injections } \\
\text { every } 2 \text { weeks }\end{array}$ \\
\hline Approval & $\begin{array}{l}\text { CAPS: } \\
\geq 8 \text { months and } \geq 10 \mathrm{~kg} \\
\text { FDA: NOMID only; EMA: all } \\
\text { CAPS phenotypes }\end{array}$ & $\begin{array}{l}\text { CAPS: } \\
\text { FDA: FCAS and MWS } \geq 12 \text { years } \\
\text { EMA: not approved }\end{array}$ & $\begin{array}{l}\text { CAPS: } \\
\text { FDA: FCAS and MWS } \geq 4 \text { years } \\
\text { EMA: all CAPS phenotypes } \geq 2 \text { years } \\
\text { SJIA: } \\
\geq 2 \text { years (EMA, FDA) }\end{array}$ & $\begin{array}{l}\text { sJIA: } \\
\geq 2 \text { years (EMA, FDA) }\end{array}$ \\
\hline $\begin{array}{l}\text { Costs per year } \\
(\text { US } \$)^{\mathrm{a}}\end{array}$ & Сa. 17,000 & Ca. 250,000 & Ca. 100,000 & Ca. 25,000 \\
\hline
\end{tabular}




\section{Pharmacologic treatment options in SJIA}

High-dosed corticosteroids (1-2 mg/kg) may quickly improve inflammatory attacks in sJIA and are still considered the gold standard in treatment initiation [51]. Due to the known severe side effects (e.g. growth retardation, infections, osteoporosis, hyperglycemia), corticosteroid treatment requires rapid dose tapering and, if needed, addition of diseasemodifying antirheumatic drugs (DMARDs) or biologics. Recently, a limited number of placebo-controlled and open-label studies revealed good efficacy of anti-IL-1 and anti-IL-6 blockers in sJIA, either as first-line monotherapy or in combination with corticosteroids [52, 53]. To date, only canakinumab (4-weekly dosing intervals in sJIA) and anti-IL-6 receptor antagonist tocilizumab are approved by EMA and FDA [52, 53]. Tocilizumab (Actemra ${ }^{\circledR}$, RoActemra ${ }^{\circledR}$ ) is a humanized anti-IL-6 receptor antibody that binds to soluble and membrane-bound IL-6 receptor and is administered in 2-weekly intervals i.v. (Table 2). Anakinra and rilonacept were effective too in small patient numbers with sJIA $[54,55]$. Of note, results from two clinical studies suggested a beneficial effect of early anakinra treatment on the clinical course of sJIA (monophasic vs. chronic intermittent or active disease) as 75-85\% of patients showed inactive disease after 1 year [32, 54].

The adverse events recorded with canakinumab and other anti-IL-1 blockers in sJIA are in line with those reported from CAPS [53]. Side effects of tocilizumab treatment in sJIA patients include increased susceptibility to infections, neutropenia, thrombopenia, hyperlipidemia and elevated transaminases [52].

Compared to CAPS, sJIA is a more heterogeneous disease. Anti-IL-1 and anti-IL-6 targeting therapies are effective in sJIA, but do not present the overwhelming complete response rates that are known from anti-IL-1 treatment in CAPS. Also, many sJIA patients treated with biologics require additional corticosteroids and/or DMARDs to achieve symptom control. Whether or not anti-cytokine therapies can prevent chronic active disease courses with progressive joint destruction and replace corticosteroids as first-line treatment is still unclear and will be subject of future studies. If the initial corticosteroid therapy does not result in adequate clinical response, the early use of canakinumab or tocilizumab is highly recommended to avoid severe disease-associated complications [51].

\section{Compliance with Ethics Guidelines}

\section{Conflict of Interest}

Hanna Bonnekoh declares no conflict of interest.

Karoline Krause declares the receipt of honoraria and research grants from Novartis, Regeneron and Roche.

\section{Human and Animal Rights and Informed Consent}

This article does not contain any studies with human or animal subjects performed by the authors. 


\section{References and Recommended Reading}

Papers of particular interest, published recently, have been highlighted as:

- Of importance

- Of major importance

1. McDermott MF, Aksentijevich I, Galon J, McDermott EM, Ogunkolade BW, Centola M, et al. Germline mutations in the extracellular domains of the $55 \mathrm{kDa}$ TNF receptor, TNFR1, define a family of dominantly inherited autoinflammatory syndromes. Cell. 1999;97(1):133-44.

2. $\quad$ Krause K, Grattan CE, Bindslev-Jensen C, Gattorno M, Kallinich T, de Koning HD, et al. How not to miss autoinflammatory diseases masquerading as urticaria. Allergy. 2012;67(12):1465-74.

This EAACI Task Force report summarizes the clinical phenotype and diagnosis of urticarial autoinflammatory diseases.

3. $\quad$ Federici S, Sormani MP, Ozen S, Lachmann HJ, Amaryan G, Woo P, et al. Evidence-based provisional clinical classification criteria for autoinflammatory periodic fevers. Ann Rheum Dis. 2015;74(5):799-805. Here, clinical criteria are reported that help differentiating between different hereditary periodic fever syndromes.

4. Lepore L, Paloni G, Caorsi R, Alessio M, Rigante D, Ruperto N, et al. Follow-up and quality of life of patients with cryopyrin-associated periodic syndromes treated with Anakinra. J Pediatr. 2010;157(2):310-5.

5. Lachmann HJ, Goodman HJ, Gilbertson JA, Gallimore JR, Sabin CA, Gillmore JD, et al. Natural history and outcome in systemic AA amyloidosis. N Engl J Med. United States: 2007 Massachusetts Medical Society. 2007;2361-71.

6.• Ter Haar N, Lachmann H, Ozen S, Woo P, Uziel Y, Modesto $\mathrm{C}$, et al. Treatment of autoinflammatory diseases: results from the Eurofever Registry and a literature review. Ann Rheum Dis. England 2013;678-85. This study presents real-life treatment response results from a big cohort of autoinflammatory patients.

7. Toplak N, Frenkel J, Ozen S, Lachmann HJ, Woo P, Kone-Paut I, et al. An international registry on autoinflammatory diseases: the Eurofever experience. Ann Rheum Dis. 2012;71(7):1177-82.

8. Giamarellos-Bourboulis EJ, Mouktaroudi M, Bodar E, van der Ven J, Kullberg BJ, Netea MG, et al. Crystals of monosodium urate monohydrate enhance lipopolysaccharide-induced release of interleukin 1 beta by mononuclear cells through a caspase 1-mediated process. Ann Rheum Dis. England 2009;273-8.

9. Masters SL, Dunne A, Subramanian SL, Hull RL, Tannahill GM, Sharp FA, et al. Activation of the NLRP3 inflammasome by islet amyloid polypeptide provides a mechanism for enhanced IL-1beta in type 2 diabetes. Nat Immunol. United States 2010;897-904.
10. Opipari A, Franchi L. Role of inflammasomes in intestinal inflammation and Crohn's disease. Inflamm Bowel Dis. United States 2015;173-81.

11. Maurer M, Magerl M, Metz M, Siebenhaar F, Weller K, Krause K. Practical algorithm for diagnosing patients with recurrent wheals or angioedema. Allergy. 2013;68(6):816-9.

12. Cuisset L, Jeru I, Dumont B, Fabre A, Cochet E, Bozec $\mathrm{JL}$, et al. Mutations in the autoinflammatory cryopyrinassociated periodic syndrome gene: epidemiological study and lessons from eight years of genetic analysis in France. 2011.

13. Maksimovic L, Stirnemann J, Caux F, Ravet N, Rouaghe $S$, Cuisset L, et al. New CIAS1 mutation and anakinra efficacy in overlapping of Muckle-Wells and familial cold autoinflammatory syndromes. Rheumatol (Oxford, Engl). England 2008;309-10.

14. Hoffman HM, Mueller JL, Broide DH, Wanderer AA, Kolodner RD. Mutation of a new gene encoding a putative pyrin-like protein causes familial cold autoinflammatory syndrome and Muckle-Wells syndrome. Nat Genet. 2001;29(3):301-5.

15. Feldmann J, Prieur AM, Quartier P, Berquin P, Certain $\mathrm{S}$, Cortis E, et al. Chronic infantile neurological cutaneous and articular syndrome is caused by mutations in CIAS1, a gene highly expressed in polymorphonuclear cells and chondrocytes. Am J Hum Genet. 2002;71(1):198-203.

16. Martinon F, Burns K, Tschopp J. The inflammasome: a molecular platform triggering activation of inflammatory caspases and processing of proIL-beta. Mol Cell. United States 2002;417-26.

17. Dinarello CA. Immunological and inflammatory functions of the interleukin-1 family. Annu Rev Immunol. 2009;27:519-50.

18. Nakamura Y, Kambe N, Saito M, Nishikomori R, Kim YG, Murakami M, et al. Mast cells mediate neutrophil recruitment and vascular leakage through the NLRP3 inflammasome in histamine-independent urticaria. J Exp Med. 2009;206(5):1037-46.

19. Ravelli A, Martini A. Juvenile idiopathic arthritis. Lancet. England 2007;767-78.

20. Danner S, Sordet C, Terzic J, Donato L, Velten M, Fischbach M, et al. Epidemiology of juvenile idiopathic arthritis in Alsace, France. J Rheumatol. Canada 2006;1377-81.

21. Berntson L, Andersson Gare B, Fasth A, Herlin T, Kristinsson J, Lahdenne P, et al. Incidence of juvenile idiopathic arthritis in the Nordic countries. A 
population based study with special reference to the validity of the ILAR and EULAR criteria. J Rheumatol. Canada 2003;2275-82.

22. Fujikawa S, Okuni M. Clinical analysis of 570 cases with juvenile rheumatoid arthritis: results of a nationwide retrospective survey in Japan. Acta Paediatr Jpn. 1997;39(2):245-9.

23. Frosch M, Roth J. New insights in systemic juvenile idiopathic arthritis-from pathophysiology to treatment. Rheumatol (Oxford, Engl). England 2008;1215.

24. Prakken B, Albani S, Martini A. Juvenile idiopathic arthritis. Lancet. England: 2011 Elsevier Ltd; 2011;2138-49.

25. Singh-Grewal D, Schneider R, Bayer N, Feldman BM. Predictors of disease course and remission in systemic juvenile idiopathic arthritis: significance of early clinical and laboratory features. Arthritis Rheum. 2006;54(5):1595-601.

26. Correll CK, Binstadt BA. Advances in the pathogenesis and treatment of systemic juvenile idiopathic arthritis. Pediatr Res. United States 2014;176-83.

27. Saha A, Chopra Y, Theis JD, Vrana JA, Sethi S. AA amyloidosis associated with systemic-onset juvenile idiopathic arthritis. Am J Kidney Dis. 2013;62(4):8348.

28. Duarte C, Gomes C, Correia AJ, Salgado M. Renal amyloidosis: an uncommon complication of juvenile idiopathic arthritis. Clin Rheumatol. 2006;25(4):5489.

29. Maeno N, Takei S, Nomura Y, Imanaka H, Hokonohara M, Miyata K. Highly elevated serum levels of interleukin-18 in systemic juvenile idiopathic arthritis but not in other juvenile idiopathic arthritis subtypes or in Kawasaki disease: comment on the article by Kawashima et al. Arthritis Rheum. 2002;46(9):2539-41.

author reply 41-2.

30. De Benedetti F, Massa M, Pignatti P, Albani S, Novick D, Martini A. Serum soluble interleukin 6 (IL-6) receptor and IL-6/soluble IL-6 receptor complex in systemic juvenile rheumatoid arthritis. J Clin Invest. 1994;93(5):2114-9.

31. de Benedetti F, Massa M, Robbioni P, Ravelli A, Burgio GR, Martini A. Correlation of serum interleukin- 6 levels with joint involvement and thrombocytosis in systemic juvenile rheumatoid arthritis. Arthritis Rheum. 1991;34(9):1158-63.

32. Pascual V, Allantaz F, Arce E, Punaro M, Banchereau J. Role of interleukin-1 (IL-1) in the pathogenesis of systemic onset juvenile idiopathic arthritis and clinical response to IL-1 blockade. J Exp Med. United States 2005;1479-86.

33. Petty RE, Southwood TR, Manners P, Baum J, Glass DN, Goldenberg J, et al. International League of Associations for Rheumatology classification of juvenile idiopathic arthritis: second revision, Edmonton, 2001. J Rheumatol. Canada 2004;390-2.
34. Levy R, Gerard L, Kuemmerle-Deschner J, Lachmann HJ, Kone-Paut I, Cantarini L, et al. Phenotypic and genotypic characteristics of cryopyrin-associated periodic syndrome: a series of 136 patients from the Eurofever Registry. Ann Rheum Dis. 2014.

35. Wittkowski H, Frosch M, Wulffraat N, GoldbachMansky R, Kallinich T, Kuemmerle-Deschner J, et al. S100A12 is a novel molecular marker differentiating systemic-onset juvenile idiopathic arthritis from other causes of fever of unknown origin. Arthritis Rheum. 2008;58(12):3924-31.

36. Lachmann HJ, Lowe P, Felix SD, Rordorf C, Leslie K, Madhoo S, et al. In vivo regulation of interleukin 1 beta in patients with cryopyrin-associated periodic syndromes. J Exp Med. 2009;206(5):1029-36.

37. Kieffer C, Cribier B, Lipsker D. Neutrophilic urticarial dermatosis: a variant of neutrophilic urticaria strongly associated with systemic disease. Report of 9 new cases and review of the literature. Medicine. 2009;88(1):2331.

38. Fortna RR, Gudjonsson JE, Seidel G, Dicostanzo D, Jacobson M, Kopelman M, et al. Persistent pruritic papules and plaques: a characteristic histopathologic presentation seen in a subset of patients with adultonset and juvenile Still's disease. J Cutan Pathol. Denmark 2010;932-7.

39. Frosch M, Metze D, Foell D, Vogl T, Sorg C, Sunderkotter C, et al. Early activation of cutaneous vessels and epithelial cells is characteristic of acute systemic onset juvenile idiopathic arthritis. Exp Dermatol. Denmark 2005;259-65.

40. Nakagawa K, Gonzalez-Roca E, Souto A, Kawai T, Umebayashi H, Campistol JM, et al. Somatic NLRP3 mosaicism in Muckle-Wells syndrome. A genetic mechanism shared by different phenotypes of cryopyrin-associated periodic syndromes. Ann Rheum Dis. 2015;74(3):603-10.

41. Barnes MG, Grom AA, Thompson SD, Griffin TA, Pavlidis $\mathrm{P}$, Itert $\mathrm{L}$, et al. Subtype-specific peripheral blood gene expression profiles in recent-onset juvenile idiopathic arthritis. Arthritis Rheum. 2009; 60(7):2102-12.

42.• Nirmala N, Grom A, Gram H. Biomarkers in systemic juvenile idiopathic arthritis: a comparison with biomarkers in cryopyrin-associated periodic syndromes. Curr Opin Rheumatol. 2014;26(5):543-52.

This study summarizes the known data of diagnostic biomarkers in CAPS and sJIA.

43. Hawkins PN, Lachmann HJ, McDermott MF. Interleukin-1-receptor antagonist in the Muckle-Wells syndrome. N Engl J Med. United States 2003;2583-4.

44. Lachmann HJ, Kone-Paut I, Kuemmerle-Deschner JB, Leslie KS, Hachulla E, Quartier P, et al. Use of canakinumab in the cryopyrin-associated periodic syndrome. N Engl J Med. United States: 2009 Massachusetts Medical Society; 2009. p. 2416-25.

45. Goldbach-Mansky R, Dailey NJ, Canna SW, Gelabert A, Jones J, Rubin BI, et al. Neonatal-onset multisystem inflammatory disease responsive to interleukin-1beta 
inhibition. N Engl J Med. United States: 2006 Massachusetts Medical Society; 2006. p. 581-92.

46. Goldbach-Mansky R, Shroff SD, Wilson M, Snyder C, Plehn S, Barham B, et al. A pilot study to evaluate the safety and efficacy of the long-acting interleukin-1 inhibitor rilonacept (interleukin-1 Trap) in patients with familial cold autoinflammatory syndrome. Arthritis Rheum. 2008;58(8):2432-42.

47. Klein AK, Horneff G. Improvement of sensoneurinal hearing loss in a patient with Muckle-Wells syndrome treated with anakinra. Klin Padiatr. 2010;222(4):266-8.

48. Kuemmerle-Deschner JB, Koitschev A, Ummenhofer K, Hansmann S, Plontke SK, Koitschev C, et al. Hearing loss in Muckle-Wells syndrome. Arthritis Rheum. 2013;65(3):824-31.

49. Enriquez R, Sirvent AE, Padilla S, Noguera-Pons R, Andrada E, Ardoy F, et al. Nephrotic syndrome and AA amyloidosis revealing adult-onset cryopyrin-associated periodic syndrome. Ren Fail. 2013;35(5):738-41.

50. Rossi-Semerano L, Fautrel B, Wendling D, Hachulla E, Galeotti C, Semerano L, et al. Tolerance and efficacy of off-label anti-interleukin-1 treatments in France: a nationwide survey. Orphanet J Rare Dis. England 2015;19.

51. Ringold S, Weiss PF, Beukelman T, DeWitt EM, Ilowite NT, Kimura Y, et al. 2013 update of the 2011 American College of Rheumatology recommendations for the treatment of juvenile idiopathic arthritis: recommendations for the medical therapy of children with systemic juvenile idiopathic arthritis and tuberculosis screening among children receiving biologic medications. Arthritis Rheum. 2013;65(10):2499-512.

52. De Benedetti F, Brunner HI, Ruperto N, Kenwright A, Wright S, Calvo I, et al. Randomized trial of tocilizumab in systemic juvenile idiopathic arthritis. N Engl J Med. 2012;367(25):2385-95.

53. Ruperto N, Quartier P, Wulffraat N, Woo P, Ravelli A, Mouy R, et al. A phase II, multicenter, open-label study evaluating dosing and preliminary safety and efficacy of canakinumab in systemic juvenile idiopathic arthritis with active systemic features. Arthritis Rheum. 2012;64(2):557-67.

54. Nigrovic PA, Mannion M, Prince FH, Zeft A, Rabinovich CE, van Rossum MA, et al. Anakinra as first-line disease-modifying therapy in systemic juvenile idiopathic arthritis: report of forty-six patients from an international multicenter series. Arthritis Rheum. 2011;63(2):545-55.

55. Lovell DJ, Giannini EH, Reiff AO, Kimura Y, Li S, Hashkes PJ, et al. Long-term safety and efficacy of rilonacept in patients with systemic juvenile idiopathic arthritis. Arthritis Rheum. 2013;65(9):2486-96. 